hep-th/0311238

PUPT-2101

\title{
On Nonperturbative Exactness of Konishi Anomaly and the Dijkgraaf-Vafa Conjecture
}

\author{
Peter Svrcek \\ Joseph Henry Laboratories, Princeton University \\ Princeton, New Jersey 08544, USA
}

\begin{abstract}
In this paper we study the nonperturbative corrections to the generalized Konishi anomaly that come from the strong coupling dynamics of the gauge theory. We consider $U(N)$ gauge theory with adjoint and $S p(N)$ or $S O(N)$ gauge theory with symmetric or antisymmetric tensor. We study the algebra of chiral rotations of the matter field and show that it does not receive nonperturbative corrections. The algebra implies Wess-Zumino consistency conditions for the generalized Konishi anomaly which are used to show that the anomaly does not receive nonperturbative corrections for superpotentials of degree less than $2 l+1$ where $2 l=3 c(A d j)-c(R)$ is the one-loop beta function coefficient. The superpotentials of higher degree can be nonperturbatively renormalized because of the ambiguities in the UV completion of the gauge theory. We discuss the implications for the Dijkgraaf-Vafa conjecture.
\end{abstract}

November 2003 


\section{Introduction}

Recently there has been a renewed interest in the study of $\mathcal{N}=1$ supersymmetric gauge theories thanks to the work of Dijkgraaf and Vafa [1], [2], [3] who used string theory arguments to calculate holomorphic data of a large class of gauge theories in terms of an auxiliary matrix model. The bosonic potential of the matrix model is the superpotential of the gauge theory. Identifying the generating function for the glueball moments with the matrix model resolvent, the effective superpotential of the gauge theory with the massive scalar integrated out was related to the planar matrix model free energy. For the $U(N)$ gauge theory, the nonperturbative part of the superpotential comes from the measure of the matrix model and is given by a sum of Veneziano-Yankielowicz superpotentials of the $U\left(N_{i}\right)$ subgroups. The perturbative part is given by a sum of planar diagrams of the matrix model.

The conjecture can be studied without recourse to string theory arguments. For a pedagogical introduction to the gauge theory methods used to study the Dijkgraaf-Vafa conjecture, see [4]. The authors of [5] gave a field theory argument showing that the Feynman diagrams contributing to the perturbative part of the glueball superpotential reduce to matrix model diagrams. A different approach was pursued in [6] using the chiral ring of the gauge theory. The generalized Konishi anomalies of the chiral rotations of the adjoint field imply constraints between chiral operators. These constraints have the same form as the loop equations of the matrix model in the planar limit. Hence the effective superpotential can be expressed in terms of the matrix model free energy up to a coupling independent term which can by seen to be a sum of Veneziano-Yankielowicz superpotentials by taking the limit of large couplings of the superpotential.

To complete the above argument it is necessary to verify that the generalized Konishi anomaly equations remain valid nonperturbatively and that the low energy effective description of the gauge theory in terms of the glueball fields is correct. In [6] it was suggested that one can prove the absence of corrections to the generalized Konishi anomaly by showing that the algebra of chiral rotations of the matter field does not have nonperturbative corrections and then arguing along the lines of Wess-Zumino consistency conditions that the anomalies do not have nonperturbative corrections. In this paper we carry out this proposal. We show that the Konishi anomaly does not have nonperturbative corrections for superpotentials of degree less than $2 l+1$ where $2 l=3 c(A d j)-c(R)$ is the one-loop beta function coefficient. 
The consistency conditions do not completely fix the nonperturbative corrections to anomaly for superpotentials of a degree higher than $2 l$. Such corrections are expected due to ambiguities in the definition of highly nonrenormalizable operators like $\operatorname{Tr} \Phi^{n}$ [7], [8] and [9]. We show that all the ambiguities can be absorbed into nonperturbative redefinition of the superpotential. There are additional UV ambiguities for gauge theories which are not asymptotically free coming the freedom in their UV completion. For these theories our proof does not apply because $\Lambda^{2 l}$ has zero or negative dimension, whence there are infinitely many types of corrections of a given dimension. The consistency conditions are not powerful enough to constrain these corrections uniquely. In summary, in this paper we prove the absence of nonperturbative corrections to the generalized Konishi anomaly that come from strong coupling dynamics and determine the form of corrections for high degree superpotentials.

The proof can be applied to gauge theories whose algebra of chiral rotations of matter fields forms an extension of a partial Virasoro algebra. For example it is possible to consider matter in other than adjoint representation. In particular we show nonrenormalization of the generalized Konishi anomaly for $S O(N)$ and $S p(N)$ gauge theories with matter in the symmetric or antisymmetric representation. The nonrenormalization of the generalized Konishi anomaly for $S p(N)$ with the antisymmetric tensor is expected in the light of recent results [10] and [7] that demonstrated agreement between the effective superpotential obtained using Konishi anomalies with the dynamically generated superpotential approach [11], [12]. The papers [10] and [7] resolved a puzzle raised in [13], [14] and [15] about the application of Dijkgraaf-Vafa correspondence for $S p(N)$ with antisymmetric matter.

\section{Organization and Results of the Paper}

In section 2 we introduce the algebra of chiral rotations of the matter field and show that it is an $\mathcal{N}=1$ extension of a partial Virasoro algebra. We consider the $U(N)$ gauge theory with adjoint scalar to keep the discussion concrete. In section 3 we discuss the generalized Konishi anomalies of the chiral rotations and use the Virasoro symmetry to derive Wess-Zumino consistency conditions for the anomalies. In section 4 we use $U(1)$ symmetries of the gauge theory to determine the form of the nonperturbative corrections. In section 5 we use the Lie algebraic structure of the algebra of chiral rotations to prove that the algebra cannot get deformed nonperturbatively. This implies that the Wess-Zumino consistency conditions derived in section 4 are exact nonperturbatively. We use them to 
show for $U(N)$ in section 6 and for $S O(N)$ and $S p(N)$ in section 7 that the generalized Konishi anomaly cannot have nonperturbative corrections except for nonperturbative renormalization of superpotentials of degree greater than $2 l=3 c(A d j)-c($ Matter $)$. In section 8 we review the loop equations of the planar matrix model, considering them as anomalies of the matrix model free energy under reparametrization of the matrix $M$ to highlight their similarity with gauge theory anomalies. In section 9 we discuss the implications of the results for the Dijkgraaf-Vafa conjecture.

\section{The Algebra of Chiral Rotations}

In [6], a series of constraints for the chiral operators were derived by considering the possible anomalies of the chiral rotations $\delta \Phi=f\left(\Phi, W_{\alpha}\right)$ of the adjoint scalar field. These chiral rotations are generated by the operators

$$
\begin{aligned}
L_{n} & =\Phi^{n+1} \frac{\delta}{\delta \Phi} \\
Q_{n, \alpha} & =\frac{1}{4 \pi} W_{\alpha} \Phi^{n+1} \frac{\delta}{\delta \Phi} \\
R_{n} & =-\frac{1}{32 \pi^{2}} W_{\alpha} W^{\alpha} \Phi^{n+1} \frac{\delta}{\delta \Phi} .
\end{aligned}
$$

The action of the operators (2.1) on the single trace chiral operators $u_{k}=\operatorname{Tr} \Phi^{k}, w_{k, \alpha}=$ $\frac{1}{4 \pi} \operatorname{Tr} W_{\alpha} \Phi^{k}$ and $r_{k}=-\frac{1}{32 \pi^{2}} \operatorname{Tr} W_{\alpha}^{2} \Phi^{k}$ is

$$
\begin{aligned}
L_{n} u_{k} & =k u_{k+n}, \\
Q_{n, \alpha} u_{k} & =k w_{k+n, \alpha},
\end{aligned}
$$

The classical commutation relations of the generators follow from the definitions (2.1)

$$
\begin{aligned}
{\left[L_{m}, L_{n}\right] } & =(n-m) L_{m+n}, \\
{\left[L_{m}, Q_{n, \alpha}\right] } & =(n-m) Q_{n+m, \alpha}, \\
{\left[L_{m}, R_{n}\right] } & =(n-m) R_{m+n}, \\
\left\{Q_{m, \alpha}, Q_{n, \beta}\right\} & =-\epsilon_{\alpha \beta}(n-m) R_{n+m}, \\
{\left[Q_{m, \alpha}, R_{n}\right] } & =0, \\
{\left[R_{m}, R_{n}\right] } & =0,
\end{aligned}
$$


where $m, n \geq-1$. The last two commutators are trivially zero in the chiral ring because the third and higher powers of $W_{\alpha}$ are chiral ring descendants. The $L_{n}$ 's form a partial Virasoro subalgebra which is extended by $Q_{n, \alpha}$ 's and $R_{n}$ 's into a partial $\mathcal{N}=1$ superVirasoro algebra.

The scalar $\Phi$ and the gauge field are in the adjoint representation of the $U(N)$ gauge group so they do not couple to the diagonal $U(1)$ gauge field. Hence shifting $W_{\alpha}$ by an anticommuting number is a symmetry of the full gauge theory [6]. If we define the field $\tilde{W}_{\alpha}=W_{\alpha}+4 \pi \psi_{\alpha}$ where $\psi_{\alpha}$ is an anticommuting c-number spinor then the generator of the shift symmetry is $\partial / \partial \psi_{\alpha}$. Hence all expressions are independent of $\psi_{\alpha}$ when expressed in terms of $\tilde{W}_{\alpha}$ and $\Phi$. The shift symmetry combines the single trace chiral operators into

$$
\tilde{r}_{k}=-\frac{1}{32 \pi^{2}} \operatorname{Tr} \tilde{W}_{\alpha}^{2} \Phi^{k}=r_{k}-\psi_{\alpha} w_{k}^{\alpha}-\frac{1}{2} \psi_{\alpha} \psi^{\alpha} u_{k}
$$

The shift symmetric generators of the chiral rotations are $L_{n}$ and

$$
\begin{aligned}
\tilde{Q}_{n, \alpha} & =\frac{1}{4 \pi} \tilde{W}_{\alpha} \Phi^{n+1} \frac{\partial}{\partial \Phi}=Q_{n, \alpha}+\psi_{\alpha} L_{n}, \\
\tilde{R}_{n} & =-\frac{1}{32 \pi^{2}} \tilde{W}_{\alpha}^{2} \Phi^{n+1} \frac{\partial}{\partial \Phi}=R_{n}-\psi_{\alpha} Q_{n}^{\alpha}-\frac{1}{2} \psi_{\alpha} \psi^{\alpha} L_{n} .
\end{aligned}
$$

Shift invariance implies that the commutation relations can be written in terms of $L_{n}, \tilde{Q}_{n, \alpha}$ and $\tilde{R}_{n}$. We find that the shift invariant commutation relations are

$$
\begin{aligned}
{\left[L_{m}, \tilde{R}_{n}\right] } & =(n-m) \tilde{R}_{m+n} \\
\left\{\tilde{Q}_{m, \alpha}, \tilde{Q}_{n, \beta}\right\} & =-\epsilon_{\alpha, \beta}(n-m) \tilde{R}_{m+n} \\
{\left[\tilde{R}_{m}, \tilde{R}_{n}\right] } & =0
\end{aligned}
$$

We did not write down the $[L, \tilde{Q}]$ and $[\tilde{Q}, \tilde{R}]$ commutators because they are contained in the $[L, \tilde{R}]$ and $[\tilde{R}, \tilde{R}]$ commutators respectively. For future reference let us show that the first and the third commutation relation in (2.6) imply the remaining relation. The first commutator contains the $[L, L],[L, Q]$ and $[L, R]$ commutators. If we expand the last commutator in $\psi_{\alpha}$, all commutators are trivially zero except for the commutator multiplying $\psi_{\alpha} \psi^{\alpha}$ which is

$$
\left[L_{m}, R_{n}\right]+\left[R_{m}, L_{n}\right]+\epsilon_{\alpha \beta}\left\{Q_{m}^{\alpha}, Q_{n}^{\beta}\right\}=0
$$

We use this equation together with the $[L, R]$ commutator to get the $\{Q, Q\}$ commutator. Hence, the first and third commutator in (2.6) contain all commutation relations of the partial $\mathcal{N}=1$ super-Virasoro algebra. 


\section{Wess-Zumino Consistency Conditions for the Generalized Konishi Anomaly}

Assume that the adjoint scalar has the tree level superpotential

$$
W(\Phi)=\sum_{i=1}^{n+1} \frac{g_{i}}{i} \operatorname{Tr} \Phi^{i} .
$$

The effective superpotential of the gauge theory is

$$
\exp \left(-\int d^{2} \theta W_{\text {eff }}\right)=\left\langle\exp \left(-\int d^{4} x d^{2} \theta W(\Phi)\right)\right\rangle
$$

where the path integral is over the massive fields in the presence of a slowly varying background gauge field. The effective superpotential has an anomaly under the chiral rotations generated by $L_{n}, Q_{n, \alpha}, R_{n}$

$$
\begin{aligned}
L_{n} W_{\text {eff }} & =\mathcal{L}_{n}, \\
Q_{n, \alpha} W_{\text {eff }} & =\mathcal{Q}_{n, \alpha}, \\
R_{n} W_{\text {eff }} & =\mathcal{R}_{n} .
\end{aligned}
$$

The perturbative anomaly of the effective superpotential under the chiral rotations $\tilde{R}_{n}$ were derived in [6]

$$
\tilde{\mathcal{R}}_{k}=\sum_{i=1}^{n+1} g_{i} \tilde{r}_{k+i}-\sum_{i=0}^{k} \tilde{r}_{i} \tilde{r}_{k-i} .
$$

The equation (3.4) is obtained from the $1 / z^{k+2}$ term of the equation (4.14) of [6] for the generating function for the generalized Konishi anomaly remembering that the $g_{i}$ in this paper is $g_{i-1}$ of [6]. The first part of $\tilde{\mathcal{R}}_{k}$ is the classical variation of the superpotential and the second part comes from the anomalous transformation of the measure of $\Phi$ under the chiral rotation. The anomalous divergence of the currents generating the chiral rotations is the Konishi anomaly

$$
\bar{D}_{\alpha} \bar{D}^{\alpha} J_{L_{n}}=\mathcal{L}_{n}
$$$$
\text { ... }
$$

Hence the generalized Konishi anomaly, being $\bar{D}_{\alpha}$ exact, is a chiral ring descendant. Setting (3.4) to zero gives nontrivial relations between the chiral operators, which enabled the authors of [6] to study the dynamics of the gauge theory and to give a partial proof of the Dijkgraaf-Vafa conjecture. We will return to this in more detail in the last section. 
The Lie algebra structure of the chiral rotations implies relations between anomalies of different chiral rotations. These conditions were first discussed by Wess and Zumino [16]. They express the closure of the Lie algebra under commutation relations. For two chiral rotations $R_{1}$ and $R_{2}$ the anomaly of the effective superpotential under $R_{1} R_{2}-R_{2} R_{1}$ must be the same as the anomaly under $R_{3}=\left[R_{1}, R_{2}\right]$

$$
R_{1} \mathcal{R}_{2}-R_{2} \mathcal{R}_{1}=\mathcal{R}_{\left[R_{1}, R_{2}\right]} .
$$

The Wess-Zumino consistency conditions for the algebra of chiral rotations (2.3) are

$$
\begin{aligned}
L_{m} \mathcal{L}_{n}-L_{n} \mathcal{L}_{m} & =(n-m) \mathcal{L}_{n+m}, \\
L_{m} \mathcal{Q}_{n, \alpha}-Q_{n, \alpha} \mathcal{L}_{m} & =(n-m) \mathcal{Q}_{n, \alpha}, \\
\ldots &
\end{aligned}
$$

In the shift invariant notation, we have

$$
\begin{aligned}
L_{m} \tilde{\mathcal{R}}_{n}-\tilde{R}_{n} \mathcal{L}_{m} & =(n-m) \tilde{\mathcal{R}}_{m+n} \\
\tilde{Q}_{m, \alpha} \tilde{\mathcal{Q}}_{n, \beta}+\tilde{Q}_{n, \beta} \tilde{\mathcal{Q}}_{m, \alpha} & =-\epsilon_{\alpha \beta}(n-m) \tilde{\mathcal{R}}_{m+n}, \\
\tilde{R}_{m} \tilde{\mathcal{R}}_{n}-\tilde{R}_{n} \tilde{\mathcal{R}}_{m} & =0 .
\end{aligned}
$$

Let us verify that the perturbative anomaly (3.4) satisfies the Wess-Zumino consistency conditions. The calculations are routine so we will check only the first equation in (3.7). Expanding (3.4) with respect to $\psi_{\alpha}$ we find using (2.4) and (2.5)

$$
\mathcal{L}_{k}=\sum_{i=1}^{n+1} g_{i} u_{k+i}-2 \sum_{i=0}^{k} u_{i} r_{k-i} .
$$

The action of $L_{k}$ on $\mathcal{L}_{l}$ is

$$
L_{k} \mathcal{L}_{l}=\sum_{i=1}^{n+1}(k+i) g_{i} u_{l+k}-2 \sum_{i=0}^{l}\left(i \tilde{u}_{i+k} r_{l-i}+(l-i) u_{i} r_{k+l-i}\right) .
$$

Subtracting from this the analogous expression for $L_{l} \mathcal{L}_{k}$ we get

$$
L_{k} \mathcal{L}_{l}-L_{l} \mathcal{L}_{k}=(l-k) \mathcal{L}_{k+l}
$$

which is the Wess-Zumino consistency condition (3.7) for the Virasoro subalgebra. 


\section{Nonpertubative Corrections}

In this section we review the argument for the absence of the multi-loop corrections to the generalized Konishi anomaly and then discuss the structure of nonperturbative corrections. For this it is instrumental to study the $U(1)$ symmetries of the gauge theory. The gauge theory has two continuous symmetries, a standard $U(1)_{R}$ symmetry and a symmetry $U(1)_{\Phi}$ under which the entire superfield $\Phi$ undergoes a rotation

$$
\Phi \rightarrow e^{i \alpha} \Phi .
$$

We also introduce a linear combination of these, $U(1)_{\theta}$, which is convenient in certain arguments. These symmetries are symmetries of the theory with nonzero superpotential if we assign nonzero $U(1)$ charges to the couplings $g_{k}$.

$\begin{array}{ccccc} & \Delta & Q_{\Phi} & Q_{R} & Q_{\theta} \\ \Phi & 1 & 1 & 2 / 3 & 0 \\ W_{\alpha} & 3 / 2 & 0 & 1 & 1 \\ g_{k} & 1-k & -k & \frac{2}{3}(1-k) & 2 \\ \Lambda^{2 l} & 2 l & 2 l & 4 l / 3 & 0 \\ \tilde{\mathcal{R}}_{k} & 6+k & k & 4+2 k / 3 & 4\end{array}$

The one-loop beta function coefficient is $2 l=3 c(A d j)-c(R)$ where $c(R)$ is the index of the representation $R$ of the matter field

$\begin{array}{cccccc}R & U_{A d j}(N) & S O(N)_{A} & S O(N)_{S} & S p(N)_{A} & S p(N)_{S} \\ c(R) & N & N-2 & N+2 & N-1 & N+1 \\ l & N & N-2 & N-3 & N+2 & N+1\end{array}$

The shift invariant $\tilde{W}_{\alpha}$ and the anticommuting shift c-number $\psi_{\alpha}$ have the same $U(1)$ charges as $W_{\alpha}$. These symmetries are violated at one loop. In the last line of the table (4.2) we have written the charges by which the anomaly $\tilde{\mathcal{R}}_{k}$ violates the $U(1)$ symmetries. The higher loop computations are finite and the $U(1)$ symmetries leave them invariant.

We are now ready to analyze the corrections to the generalized Konishi anomaly (3.4). The corrections must have the same $U(1)$ charges as $\tilde{\mathcal{R}}_{k}$. They are polynomial in the chiral operators. Furthermore, the corrections that depend on $g_{k}$ must vanish for the theory with zero superpotential and the nonperturbative corrections that depend on $\Lambda^{2 l}$ vanish when we take the strong coupling scale $\Lambda$ to zero. Hence, the corrections to the anomaly are also polynomial in $g_{k}$ and $\Lambda^{2 l}$. Referring to the table (4.2) we see that the only polynomials in $g_{k}, \Phi$ and $W_{\alpha}$ with the quantum numbers of $\tilde{\mathcal{R}}_{k}$ are the ones already present in the one loop 
expression (3.4). Hence the anomaly does not have higher loop contributions, as claimed at the end of the previous paragraph. The nonperturbative corrections are polynomial in $\Lambda^{2 l}$. The possible $j$ instanton corrections to $\tilde{\mathcal{R}}_{k}$ are of the form $\Lambda^{2 j l} g_{i+2 j l} \tilde{r}_{k+i}$ and $\Lambda^{2 j l} \tilde{r}_{i-2 j l} \tilde{r}_{k-i}$.

We can similarly derive the possible form of corrections to the extended Virasoro algebra (2.3). The corrections to the $[L, L]$ commutator are linear the Virasoro generators $L_{n}$ and polynomial in $g_{k}$ and $\Lambda^{2 h}$. The Virasoro generator $L_{n}$ (2.1) increases the $U(1)$ charges of a chiral operators by the same value as multiplication by $\Phi^{n}$. Hence, the commutator $\left[L_{m}, L_{n}\right]$ fixes $Q_{\theta}$ and increases the dimension by $m+n$. Consulting the table (4.2) we see that $g_{i}$ has $Q_{\theta}=2$ charge so the there are no corrections that depend on the superpotential. The nonperturbative $l$ instanton corrections have the form $\Lambda^{2 j l} L_{m+n-2 j l}$. Similar corrections contribute to the $[L, Q],[L, R]$ and $Q, Q$. The commutators that shift $Q_{\theta}$ by two can also have corrections proportional to $g_{i}$. Counting the $U(1)$ charges we see that the $\left[L_{m}, R_{n}\right]$ commutator has corrections $\Lambda^{2 j l} R_{m+n-2 j l}$ and $\Lambda^{2 j l} g_{i} L_{m+n+i-2 j l}$. There are similar corrections to $\{Q, Q\}$. The $[Q, R]$ and $[R, R]$ commutators cannot have corrections because they map chiral operators into chiral ring descendants.

\section{Nonrenormalization of the Algebra of Chiral Rotations}

In this section we prove the nonrenormalization of the algebra (2.3) of chiral rotations of the $U(N)$ adjoint scalar. Firstly we analyze in detail the corrections to the partial Virasoro subalgebra

$$
\left[L_{m}, L_{n}\right]=(n-m) L_{m+n}+\sum_{j>0} \Lambda^{2 j N} b_{m, n}^{j} L_{m+n-2 j N}
$$

where the coefficients $b_{m, n}^{j}$ are antisymmetric in $m$ and $n$ by antisymmetry of the commutator (5.1). The coefficient $b_{m, n}^{j}$ is in front of $L_{m+n-2 j N}$ hence it vanishes if $m+n-2 j N<-1$ because $L_{-1}$ is the lowest nonzero generator. We will prove that all the coefficients $b_{m, n}^{j}$ can be absorbed into nonperturbative redefinition of the Virasoro generators

$$
\mathrm{L}_{n}=L_{n}+\sum_{j>0} a_{n}^{j} \Lambda^{2 j N} L_{n-2 j N}
$$

where $a_{n}^{j}$ is the coefficient of the $j$-instanton correction to $\mathrm{L}_{n}$. The Virasoro generators are corrected which is natural considering that they act on the nonperturbatively corrected 
chiral operators $\tilde{r}_{k}$. In terms of the new basis of generators $\mathrm{L}_{n}$ the commutations relations of the partial Virasoro algebra remain valid nonperturbatively

$$
\left[\mathrm{L}_{m}, \mathrm{~L}_{n}\right]=(n-m) \mathrm{L}_{m+n}
$$

Calculating the coefficients $a_{n}^{j}$ which parameterize the nonperturbative corrections to $\mathrm{L}_{n}$ 's is beyond the scope of the present paper. We will show instead that there is a choice of $a_{n}^{j}$ 's for which the Virasoro algebra takes the standard form (5.3). This shows that the algebra itself is not corrected even though the Virasoro operators might receive corrections. We make induction in the instanton number of the nonperturbative corrections. The coefficients $b_{m, n}^{j}$ obey equations that follow from the Jacobi identity

$$
\left[L_{l},\left[L_{m}, L_{n}\right]\right]+\left[L_{n},\left[L_{l}, L_{m}\right]\right]+\left[L_{m},\left[L_{n}, L_{l}\right]\right]=0
$$

On the zero instanton level the identity reduces to the Jacobi identity for the Virasoro algebra which is satisfied. On the one instanton level, we evaluate the commutators in (5.4) using (5.1) to find the coefficient of the $\Lambda^{2 N} L_{l+m+n-2 N}$ term which has to be zero

$$
(n-m) b_{l, m+n}^{1}+(m+n-l-2 N) b_{m, n}^{1}+\text { cyclic permutations }=0 .
$$

The one instanton corrections can be absorbed into one instanton corrections to $L_{n}$ 's (5.2). The new commutation relations are

$$
\left[\mathrm{L}_{m}, \mathrm{~L}_{n}\right]=(n-m) \mathrm{L}_{m+n}+\mathrm{b}_{m, n}^{1} \Lambda^{2 N} \mathrm{~L}_{m+n-2 N}+\ldots
$$

where $b_{\mathrm{m}, \mathrm{n}}^{1}$ 's are the redefined nonperturbative corrections

$$
\mathrm{b}_{m, n}^{1}=b_{m, n}^{1}+(n-m-2 N) a_{n}^{1}+(n-m+2 N) a_{m}^{1}-(n-m) a_{m+n}^{1}
$$

We show that $b_{m, n}^{1}$ can be set to zero by redefinition $\mathrm{L}_{m+n}=L_{m+n}+a_{m+n}^{1} \Lambda^{2 N} \mathrm{~L}_{m+n-2 N}$ by induction on $m+n$. The first step of the induction holds because $b_{m, n}^{1}$ vanishes for $m+n<2 N-1$. By induction hypothesis we assume that we have redefined $L_{m+n}$ for $m+n<M$ so that $b_{m, n}^{1}=0$. Setting $l, m, n$ in equation (5.5) equal to $0, m, M-m$ respectively, we find for $0<m<M$

$$
(M-2 m) b_{0, M}^{1}+(M-2 N) b_{m, M-m}^{1}+(m-M) b_{m, M-m}^{1}+m b_{M-m, m}^{1}=0 .
$$


Using antisymmetry of $b_{m, n}^{1}$ in $m$ and $n$ we rewrite this as

$$
2 N b_{m, M-m}^{1}=(M-2 m) b_{0, M}^{1}
$$

From (5.7) the redefined nonperturbative corrections are

$$
\begin{aligned}
\mathrm{b}_{0, M}^{1} & =b_{0, M}^{1}-2 N a_{M}^{1}, \\
\mathrm{~b}_{m, M-m}^{1} & =b_{m, M-m}^{1}-(M-2 m) a_{M}^{1} .
\end{aligned}
$$

We see from (5.9) that taking $a_{M}=b_{0, M}^{1} / 2 N$ we set $\mathrm{b}_{m, n}^{1}=0$ for $m+n=M$. This completes the induction in $m+n$ and shows that there are no one instanton corrections to the Virasoro algebra. We can now proceed with the induction in the instanton number by assuming absence of nonperturbative corrections to the Virasoro algebra for instanton number less than $k$. We also assume that we have redefined the the Virasoro operators $\mathrm{L}_{n}$ up to instanton number $k-1$ to set $b_{m, n}^{j}=0$ for $j<k$. The proof that the $k$ instanton corrections to the Virasoro algebra can be absorbed into $k$ instanton redefinition of the operators $\mathrm{L}_{n}$ goes exactly as the above calculation in the one instanton case because the necessary equations at the $\Lambda^{2 k N}$ order are identical to the equations $(5.5),(5.7)-(5.10)$ we found at $\Lambda^{2 N}$ order after substituting $N$ for $k N$ in all equations. The additional terms in (5.5) and (5.7) that would come from lower instanton corrections vanish by the induction hypothesis.

Now it remains to show that the commutation relations of $\mathrm{L}_{-1}=L_{-1}$ with $\mathrm{L}_{n}$ do not get corrected. Firstly consider one instanton corrections. Notice that $b_{-1,0}^{1}$ vanishes on dimensional grounds as noted below (5.1). Taking $l, m, n$ in (5.5) to be $-1,0, n$ for $n>0$ we find $2 N b_{1, n}^{1}=0$ which completes the proof of the absence of one instanton corrections. We prove the absence of $k$ instantons corrections the same way after substituting $N$ for $k N$ in $(5.5)$.

We give two different proofs of the nonrenormalization of the remaining commutators of the algebra of chiral rotations. The first one is simpler and uses the shift symmetry of the commutations relations. The second one does not use the $U(1)$ shift symmetry and hence is applicable for the $S O(N)$ and $S p(N)$ gauge theories as well. We postpone it to the Appendix A because it is more technical. From now on we do not use roman font to distinguish the nonperturbatively defined generators.

Let us outline the first argument. We use shift symmetry to fix the nonperturbative definitions $\mathrm{Q}_{n, \alpha}, \mathrm{R}_{n}$ for $n \geq 2 N$ using the nonperturbatively defined $L_{n}$ (5.2). The last commutator in (2.6)

$$
\left[\tilde{R}_{m}, \tilde{R}_{n}\right]=0
$$


cannot receive nonperturbative corrections. Its lowest $\psi_{\alpha}$ component is the $[\mathrm{R}, \mathrm{R}]=0$ commutator which has to vanish in the chiral ring because the commutator shifts $\Phi$ by a chiral operator containing the fourth power of $W_{\alpha}$. But the third and higher powers of $W_{\alpha}$ are chiral ring descendants, so the commutator has trivial action in the chiral ring. The nonperturbative corrections to the first commutator in (2.6) that are allowed by shift symmetry are

$$
\left[L_{m}, \tilde{R}_{n}\right]=(n-m) \tilde{R}_{m+n}+\sum_{j=1}^{\infty} \sum_{i=1}^{n+1} \Lambda^{2 j N} g_{i} c_{m, n}^{i, j} L_{m+n+i-2 j N}
$$

because the $\psi_{\alpha}^{2}$ component of (5.12) is the $[L, L]$ commutator, which does not have nonperturbative corrections. The nonperturbative corrections (5.12) contribute to the $[L, R]$ commutator only. To prove that these corrections vanish we evaluate the $L, Q, R$ Jacobi identity

$$
\begin{array}{r}
{\left[Q_{l, \alpha},\left[L_{m}, R_{n}\right]\right]+\left[L_{m},\left[R_{n}, Q_{l, \alpha}\right]\right]+\left[R_{n},\left[Q_{l, \alpha}, L_{m}\right]\right]=} \\
=\left[Q_{l, \alpha}, \sum_{j=1}^{\infty} \sum_{i=1}^{n+1} \Lambda^{2 j N} g_{i} c_{m, n}^{i, j} L_{m+n+i-2 j N}\right]= \\
=\sum_{j=1}^{\infty} \sum_{i=1}^{n+1} \Lambda^{2 j N} g_{i}(m+n-l+i-2 j N) c_{m, n}^{i, j} Q_{m+n+i+l-2 j N, \alpha}=0 .
\end{array}
$$

In simplifying (5.13) we used the $[L, Q]$ commutator (5.12) which is nonrenormalized by shift symmetry and the $[R, Q]=0$ commutator. Clearly, the only way to satisfy the Jacobi identity (5.13) is that $c_{m, n}^{i, j}=0$. All corrections to (5.12) vanish. Hence, none of the commutation relations of the extended Virasoro algebra get nonperturbative corrections because as we noted below 2.6 the above two commutators imply the remaining one.

\section{Nonperturbative Corrections to the Konishi Anomaly for $U(N)$ Gauge Theory}

Let us now consider nonperturbative corrections to the anomaly. The anomaly $\tilde{\mathcal{R}}_{k}$ (3.4) differs from its perturbative value implicitly through the dependence of the chiral operators $\tilde{r}_{k}$ on nonperturbative physics. In this section we ask the question whether there are additional nonperturbative corrections that depend explicitly on $\Lambda^{2 j N}$. We can easily 
introduce terms proportional to $\Lambda^{2 j N}$ into the expression for $\mathcal{R}_{k}$ by redefining the chiral operators

$$
\tilde{r}_{k}=\tilde{r}_{k}+\alpha \Lambda^{2 N} \tilde{r}_{k-2 N}+\ldots
$$

Notice that $r_{k}$ for $k>1$ are nonrenormalizable operators so their value depends on the renormalization scheme. It is natural to expect terms of the form (6.1) to relate the definitions of $r_{k}$ coming from different renormalization schemes. Hence we expect that the anomaly has generically terms proportional to $\Lambda^{2 j N}$ if we take some arbitrary prescription for $\tilde{r}_{k}$.

However, there is a natural definition of the higher moments $\tilde{r}_{k}$. In the previous section we showed that there is a preferred basis for the generators of the chiral rotations $\tilde{R}_{k}$ in terms of which the partial super-Virasoro algebra takes the standard form (2.6). We can use their action on the chiral operators to give a nonperturbative definition of nonrenormalizable operators $\tilde{r}_{k}$ for $k>1$ in terms of the the first moment $\tilde{r}_{k}=L_{k} \tilde{r}_{1}$. It follows from the commutation relations (2.6) that remaining operators $\tilde{R}_{k}$ act on the chiral operators as before (2.2).

Having defined $\tilde{r}_{k}$ nonperturbatively, we can now show using the Wess-Zumino consistency conditions that the one-loop anomaly $\sum \tilde{r}_{i} \tilde{r}_{k-i}$ in the path integral measure for $\Phi$ does not have nonperturbative corrections. We will also show that the consistency conditions allow nonperturbative renormalization of the superpotential. The consistency conditions of the full gauge theory (3.8) do not have nonperturbative corrections because their derivation rested only on the commutation relations of the super-Virasoro algebra (2.6) which are nonrenormalized. We deduced in section 4 using $U(1)$ symmetries that the general form of nonperturbative corrections to $\tilde{\mathcal{R}}_{n}$ is

$$
\begin{aligned}
\tilde{\mathcal{R}}_{k}= & \sum_{i}\left(g_{i}+\Lambda^{2 N} g_{i+2 N} c_{k, i}^{1}+\ldots\right) \tilde{r}_{k+i}+ \\
& -\sum_{i=0}^{k} \tilde{r}_{i} \tilde{r}_{k-i}-\Lambda^{2 N} \sum_{i=2 N}^{k} d_{k, i}^{1} \tilde{r}_{i-2 N} \tilde{r}_{k-i}+\ldots
\end{aligned}
$$

In writing (6.2) we take $g_{k}=0$ for $k<1$ and $k>n+1$ to simplify the notation. We can consider the corrections to the the superpotential separately from the corrections to the one-loop anomaly. The corrections to the superpotential are proportional to $\Lambda^{2 j N} g_{i+2 j N}$ which have the same quantum numbers as $\tilde{r}_{-i}$ which does not exist. Hence the two types of corrections do not mix. 
Firstly we show that all the nonperturbative corrections to the one-loop part of $\tilde{\mathcal{R}}_{k}$ vanish. Notice, that the lowest dimensional correction is $\tilde{r}_{0} \tilde{r}_{0} \Lambda^{2 N}$ which contributes to $\tilde{\mathcal{R}}_{2 N}$, hence the one-loop parts $\tilde{\mathcal{R}}_{k}$ for $k=-1,0, \ldots, 2 N-1$ does not have nonperturbative corrections. The first consistency condition (3.8) with $m=0$ simplifies to $L_{0} \tilde{\mathcal{R}}_{k}=k \tilde{\mathcal{R}}_{k}$ because $R_{k} \tilde{r}_{0} \tilde{r}_{0}=0$. In other words $L_{0}$ acting on $\tilde{\mathcal{R}}_{k}$ gives $k$ times the anomaly. But $L_{0}$ acting on a $j$-instanton correction $\Lambda^{2 j N} \tilde{r}_{i-2 j N} \tilde{r}_{k-i}$ gives back $k-2 j N$ multiple of the correction, whence all nonperturbative corrections to the one-loop part of the anomaly vanish.

It remains to consider the corrections to the classical part of $\tilde{\mathcal{R}}_{n}$. We find from (6.2) that the first consistency condition (3.8) becomes

$$
\begin{aligned}
L_{k} \tilde{\mathcal{R}}_{l}-\tilde{R}_{l} \mathcal{L}_{k}= & (l-k) \tilde{\mathcal{R}}_{l+k} \\
& +\sum_{j \geq 1} \Lambda^{2 j N} \sum_{i=-2 j N}^{n-2 j N}\left[(l+1) c_{l, i}^{j}-(k+1) c_{k, i}^{j}-(l-k) c_{k+l, i}^{j}\right] g_{i+2 j N} \tilde{r}_{k+l} .
\end{aligned}
$$

But the Wess-Zumino consistency conditions do not have nonperturbative corrections whence we set the terms in the square brackets to zero

$$
(l+1) c_{l, i}^{j}-(k+1) c_{k, i}^{j}=(l-k) c_{k+l, i}^{j} .
$$

Taking $l=0$ we have $c_{k, i}^{j}=c_{0, i}^{j}$. Clearly, this solves all the constraints coming from (6.4). Notice that the terms $\Lambda^{2 j N} c_{-1, i}^{j} \tilde{r}_{i-1}$ in $\tilde{\mathcal{R}}_{-1}$ are absent for $i<1$ because $\tilde{r}_{k} \sim \operatorname{Tr} \tilde{W}^{2} \Phi^{k}$ is defined only for positive $k$. Hence $c_{k, i}^{j}=0$ for $i<1$. In conclusion, the general form of the anomaly is

$$
\tilde{\mathcal{R}}_{k}=\sum_{i=1}^{n+1} \mathrm{~g}_{i} \tilde{r}_{k+i}-\sum_{i=0}^{k} \tilde{r}_{i} \tilde{r}_{k-i}
$$

where

$$
\mathrm{g}_{i}=g_{i}+\Lambda^{2 N} c_{0, i}^{1} g_{i+2 N}+\Lambda^{4 N} c_{0, i}^{2} g_{i+4 N}+\ldots
$$

are the nonperturbatively renormalized coefficients of the superpotential. Hence, all corrections to the classical part of the anomaly allowed by the Wess-Zumino consistency conditions can be absorbed into nonperturbative renormalization of the superpotential

$$
W(\Phi)=\sum_{i=1}^{n+1} \frac{\mathrm{g}_{i}}{i} \operatorname{Tr} \Phi^{i} .
$$


The superpotentials of degree less than $2 N+1$ cannot have noperturbative corrections. This is the only ambiguity that is not fixed by the consistency conditions. We could have anticipated it from the observation that both $g_{i}$ and $\Lambda^{2 N}$ are invariant under the chiral rotations hence substituting for $g_{i}$ any polynomial $g_{i}\left(g_{k}, \Lambda^{2 N}\right)$ with the correct quantum numbers cannot spoil the Wess-Zumino consistency conditions whose validity depends only on the Lie algebraic structure of the chiral rotations. As noted around (6.1) the nonperturbative corrections depend on the scheme used to define the single trace operators $\tilde{r}_{k}$. Using a different UV completion of the gauge theory changes the definition of the chiral operators hence it redefines the superpotential. For further discussion of Dijkgraaf-Vafa conjecture for high degree superpotentials, see [7] [8] and [9].

\section{7. $S O(N)$ and $S p(N)$ Gauge Theories with Symmetric or Antisymmetric Matter}

In this section we show that the previous analysis applies with minor modifications to the $S O(N)$ and $S p(N)$ gauge theories. It follows that the generalized Konishi anomaly in these gauge theories does not have nonperturbative corrections for superpotentials of degree less than $2 l+1$. Superpotentials of higher degree might get nonperturbatively renormalized.

The gauge group do not have a decoupled diagonal $U(1)$ subgroup hence based on the shift symmetry do not carry over from the $U(N)$ case. That is the main reason why we gave a separate proof of the nonrenormalizability of the extended Virasoro algebra which did not use shift symmetry. For simplicity, we do not consider the fermionic generators and chiral operators. The $S O(N)$ adjoint can be represented by an $N \times N$ antisymmetric matrix $\Phi^{\mathrm{T}}=-\Phi$. The gauge field transforms in the adjoint representation hence it is antisymmetric as well $W_{\alpha}^{\mathrm{T}}=-W_{\alpha}$. The $S p(N)$ has adjoint which can be represented as $2 N \times 2 N$ matrix that satisfies the condition $\Phi^{\mathrm{T}}=-J \Phi J^{-1}$ where $J$ is the invariant antisymmetric tensor of $S p(N)$. A matrix in the adjoint representation of $S p(N)$ can be written as a product of a symmetric matrix $S$ and the invariant tensor $\Phi=S J$, which explains why this representation is called symmetric in the literature. The single trace chiral operators for both gauge groups are $u_{2 k}$ and $r_{2 k}$ because the remaining chiral operators vanish by antisymmetry. Hence the odd coefficients of the superpotential (3.1) vanish $g_{2 k+1}=0$. Similarly the nonvanishing generators of the algebra of chiral rotations are $L_{2 k}$ and $R_{2 k}$ which form a closed subalgebra of the partial $\mathcal{N}=1$ super-Virasoro algebra (2.3). Our method also applies to the symmetric tensor $\Phi^{\mathrm{T}}=\Phi$ of $S O(N)$ and the antisymmetric 
tensor $\Phi^{\mathrm{T}}=J \Phi J^{-1}$ of $S p(N)$. The definitions of the representations do not restrict the chiral operators nor the chiral rotations.

The generalized Konishi anomaly for the $S O(N)$ and $S p(N)$ gauge theories has been derived in [17, 15] and [14]

$$
\begin{aligned}
\mathcal{L}_{k} & =\sum_{i=1}^{n+1} g_{i} u_{i+k}-\sum_{i=0}^{k} u_{i} r_{k-i}+c_{k}(R) r_{k} \\
\mathcal{R}_{k} & =\sum_{i=1}^{n+1} g_{i} r_{i+k}-\frac{1}{2} \sum_{i=0}^{k} r_{i} r_{k-i}
\end{aligned}
$$

where $c_{k}(R)$ depends on the representation $R$ of the matter field

$$
\begin{array}{ccccc}
R & S O_{A}(N) & S O_{S}(N) & S p_{A}(N) & S p_{S}(N) \\
c_{k}(R) & 2 & -k-1 & k+1 & -2 .
\end{array}
$$

In section 5 we proved that the algebra generated by $L_{k}$ 's and $R_{k}$ 's where $k \geq-1$ does not get renormalized. This is the algebra for symmetric $S O(N)$ and antisymmetric $S p(N)$ matter, hence the algebra of chiral rotations of these gauge theories does not receive nonperturbative corrections. The proof for the adjoint representation works exactly as before if we substitute for all subscripts of the generators in the equations of section 5 twice their value. The proof of the nonrenormalization of the $\mathcal{R}_{k}$ anomaly also carries over because the only difference in the anomaly compared to the $U(N)$ gauge theory is the $c_{k} r_{k}$ term in $\mathcal{L}_{k}$ which has the same form as $u_{0} r_{k}$ so it cannot receive corrections. The proof for $\mathcal{L}_{k}$ follows the same pattern but instead of using the Wess-Zumino consistency condition coming from $[L, R]$ commutator we use the condition coming from $[L, L]$ commutator.

\section{Virasoro Constraints for the One-Matrix Model}

In this section we review the exact constraints for the planar level free energy $F_{m}$ of the one-matrix model [18,19.] We consider the $U(N)$ matrix model that is related to the $U(N)$ gauge theory with the adjoint scalar. The $S O(N)$ and $S p(N)$ matrix models are treated similarly. We derive the loop equations by considering the Virasoro algebra of redefinitions of the matrix $M$. This highlights the similarity of the algebraic structure of the loop equations with the gauge theory anomalies. The partition function of the matrix model is

$$
Z_{m}=\exp \left(-\frac{\hat{N}^{2}}{g_{m}^{2}} F_{m}\right)=\int d^{\hat{N}^{2}} M \exp \left(-\frac{\hat{N}}{g_{m}} W(M)\right)
$$


where $W(M)=\sum_{i=1}^{n+1} \frac{g_{i}}{i} \operatorname{Tr} M^{i}$ is the potential of the matrix model and $F_{m}$ is the matrix model free energy. The partition function is invariant under arbitrary redefinition of the integration variable $M \rightarrow f(M)$. These redefinitions are symmetries of the matrix model. The generators of the redefinitions annihilate the partition function and the free energy

$$
R_{m, k}=M^{k+1} \frac{\delta}{\delta M}
$$

They form a partial Virasoro algebra

$$
\left[R_{m, k}, R_{m, l}\right]=(l-k) R_{m, k+l},
$$

where $k, l \geq-1$. Acting with $\epsilon R_{m, k}$ on the free energy $F_{m}$ we obtain the following identity

$$
\begin{aligned}
0 & =\epsilon R_{m, k} F_{m} \equiv \epsilon \mathcal{R}_{m, k} \\
& =-\frac{g_{m}^{2}}{\hat{N}^{2} Z_{m}} \delta \int d\left(M+\epsilon M^{n+1}\right) \exp \left(-\frac{\hat{N}}{g_{m}} \sum_{i=1}^{n+1} \frac{g_{i}}{i} \operatorname{Tr}\left(M+\epsilon M^{k+1}\right)^{i}\right) .
\end{aligned}
$$

Expanding (8.4) to first order in $\epsilon$ we have

$$
\mathcal{R}_{m, k}=\frac{-g_{m}^{2}}{\hat{N}^{2} Z_{m}} \int d M\left(-\frac{\hat{N}}{g_{m}} \sum_{i=1}^{n+1} g_{i} \operatorname{Tr} M^{i+k}+\operatorname{Tr} \frac{\delta M^{k+1}}{\delta M}\right) \exp \left(-\frac{\hat{N}}{g_{m}} W(M)\right)
$$

To evaluate the Jacobian we write

$$
\begin{aligned}
\operatorname{Tr} \frac{\delta M^{n+1}}{\delta M} & =\frac{\delta M_{i j}^{k+1}}{\delta M_{i j}}=\sum_{i=l}^{k} \frac{\left(M^{l} \delta M M^{k-l}\right)_{i j}}{\delta M_{i j}} \\
& =\sum_{l=0}^{k} M_{i l}^{l} \frac{\delta M_{l m}}{\delta M_{i j}} M_{m j}^{k-l}=\sum_{l=0}^{k} \operatorname{Tr} M^{l} \operatorname{Tr} M^{k-l}
\end{aligned}
$$

Hence the variation of the free energy is

$$
\mathcal{R}_{m, k}=R_{m, k} F_{m}=\sum_{i=1}^{n+1} g_{i}\left\langle\operatorname{Tr} M^{i+k}\right\rangle-\sum_{i=0}^{k}\left\langle\operatorname{Tr} M^{i} \operatorname{Tr} M^{k-i}\right\rangle
$$

In the large $\hat{N}$ limit the expectation values of products $U(N)$ invariant operators factorize $\left\langle\operatorname{Tr} M^{i} \operatorname{Tr} M^{k-i}\right\rangle=\left\langle\operatorname{Tr} M^{i}\right\rangle\left\langle\operatorname{Tr} M^{k-i}\right\rangle$. Defining $r_{m, k}=\frac{g_{m}}{\hat{N}}\left\langle\operatorname{Tr} M^{k}\right\rangle$ we rewrite (8.7) in the large $\hat{N}$ limit as

$$
\mathcal{R}_{m, k}=\sum_{i=1}^{n+1} g_{i} r_{m, i+k}-\sum_{i=0}^{k} r_{m, i} r_{m, k-i}
$$


which takes the same form as the as the Konishi anomaly (3.4). The loop equations are obtained by setting $\mathcal{R}_{m, k}=0$. They are recursion relations for $r_{m, k}$ in terms of the first $n$ moments $r_{m, 0}, \ldots, r_{m, n-1}$. Equivalently, the loop equations determine the matrix model curve $y^{2}(z)=W^{\prime 2}(z)+f(z)$ where $y(z)=\frac{g_{m}}{\hat{N}}\left\langle\operatorname{Tr} \frac{1}{z-M}\right\rangle$ is the resolvent. The consistency conditions for $\mathcal{R}_{m, k}$ are derived the same way as for the gauge theory (3.6)

$$
R_{m, k} \mathcal{R}_{m, l}-R_{m, l} \mathcal{R}_{m, k}=(l-k) \mathcal{R}_{m, k+l}
$$

It is easy to verify that (8.8) satisfies the consistency conditions (8.9). Similarly one can show that the full matrix model loop equations (8.7) satisfy (8.9). The answer (8.8) is exact in the planar limit of the matrix model, hence we do not need the consistency conditions in this paper.

\section{Implications for the Dijkgraaf-Vafa conjecture}

Let us discuss the implications of the above results for the relation between the matrix models and the supersymmetric gauge theories. We will consider the $U(N)$ gauge theory with adjoint matter to keep the discussion concrete. The anomalous variation of the free energy of the gauge theory under $R_{k}$ (3.4) has the same form as the variation of the matrix model free energy under $R_{m, k}(8.2)$ if we identify the expectation values [6]

$$
r_{k}=r_{m, k}
$$

The equations (3.4) $\tilde{R}_{k}=0$ can be considered as recursion relations for higher moments $\tilde{r}_{i}$ in terms of the first $n$ moments $\tilde{r}_{0}, \tilde{r}_{1} \ldots \tilde{r}_{n-1}$. Hence it is enough to identify the first $n$ moments in (9.1). The matrix model then determines the expectation values of all chiral operators $r_{i}$. The expectation values of the moments of the scalar depend also on the gauge symmetry breaking pattern $U(N) \rightarrow \otimes_{i=1}^{r} U\left(N_{i}\right)$ [20. The $U(1)$ photinos of the $U\left(N_{i}\right)$ subgroups can have arbitrary vacuum expectation value. These values determine all moments of the gaugino field $\operatorname{Tr} \Phi^{k} W_{\alpha}$ [21]. Hence the isolated massive vacua come with a $2 r$-dimensional fermionic moduli space where $r$ is the rank of the low energy gauge group. In conclusion, matrix model determines the expectation values of all chiral operators up to the choice of the gauge symmetry breaking pattern and $k$ independent expectation values of the $U(1)$ photino condensates. 
The generalized Konishi anomaly can be viewed as the equation of the curve

$$
y^{2}=W^{\prime 2}(z)+f(z)
$$

where $y$ is the generating function of the glueball moments [6]. This curve is identified with the matrix model curve using (9.1) which is the same as identifying the polynomials $f(z)=f_{m}(z)$. The results from section 6 on nonperturbative corrections to the Konishi anomaly imply that the gauge theory curve does not have nonperturbative deformations for superpotentials of degree less than $2 N+1$. Hence for these superpotentials the curve of the full gauge theory agrees with matrix model curve. For higher degree of the superpotential the curve can get deformed. We have identified that the only possible deformation of the curve is the nonperturbative renormalization of the superpotential. This is so essentially because the form of the curve is uniquely fixed from the Virasoro symmetry and we know from section 5 that the extended Virasoro symmetry is exact in the full gauge theory. For given $f(z)=f_{m}(z)$, the coefficients of the superpotential are the only parameters of the curve.

The effective superpotential and the matrix model free energy are generating functions for chiral operators and for the moments of $M$ respectively

$$
\begin{aligned}
\frac{\partial}{\partial g_{k}} W_{e f f} & =\left\langle\frac{\operatorname{Tr} \Phi^{k}}{k}\right\rangle, \\
\frac{\partial F_{m}}{\partial g_{k}} & =\left\langle\frac{\operatorname{Tr} M^{k}}{k}\right\rangle .
\end{aligned}
$$

To relate $W_{\text {eff }}$ and $F_{m}$, we use shift symmetry to generalize the first equation in (9.3) to a generating function for $\operatorname{Tr} \tilde{W}^{2} \Phi^{k}$. The effective superpotential is invariant under shift symmetry so it can be written as

$$
W_{\text {eff }}=\int d^{2} \psi \mathcal{F}\left(\tilde{r}_{i}\right)
$$

for some function $\mathcal{F}$. We use (9.4) to rewrite the first equation in (9.3) as

$$
\frac{\partial}{\partial g_{k}} \mathcal{F}=\left\langle\frac{\tilde{r}_{k}}{k}\right\rangle .
$$

Hence we have the relation [6]

$$
F_{m}\left(S_{i}, g_{k}\right)=\left.\mathcal{F}\left(\tilde{S}_{i}, g_{k}\right)\right|_{\psi=0}+\left.\mathcal{H}\left(\tilde{S}_{i}\right)\right|_{\psi=0}
$$


where $\mathcal{H}\left(\tilde{S}_{i}\right)$ is a coupling independent function. Similar relations for the $S p(N)$ and $S O(N)$ gauge theory are given in [14] and [7]. The derivation of the relation (9.6) rests on the Konishi anomaly equations and on the validity of low energy description of the gauge theory in terms of the glueball fields $S_{i}$. The nonrenormalization of the Konishi anomaly implies that $\mathcal{F}$ does not have additional nonperturbative corrections, whence the relation (9.6) is valid nonperturbatively. The derivation of the nonperturbative exactness of the Konishi anomaly is the first step in a full proof of the Dijkgraaf-Vafa correspondence.

\section{Acknowledgements}

I am very grateful to F. Cachazo, G. Ferretti, N. Seiberg and especially to E. Witten for useful discussions/correspondence. This research is supported in part by NSF grants PHY9802484 and PHY-0243680. Any opinions, findings and conclusions or recommendations expressed in this material are those of the authors and do not necessarily reflect the views of the National Science Foundation.

\section{Appendix A. Second Proof of the Nonrenormalization of the Algebra of Chiral Rotations}

In this appendix we give a proof of absence of nonperturbative corrections to the extended Virasoro algebra without using the shift symmetry. This proof is applicable to $S O(N)$ and $S p(N)$ gauge theories which do not posses shift symmetry. We assume from section 5 the nonrenormalization of the Virasoro subalgebra generated by $L_{n}$ 's because we did not use shift symmetry to prove it. We use the nonperturbatively defined Virasoro generators $L_{n}$ to fix the nonperturbative definition of the remaining generators by recursively commuting $Q_{n, \alpha}$ and $R_{n}$ with the raising operator $L_{1}$. Having defined the generators, let us show that the nonperturbative corrections to the $[L, Q]$ commutator vanish

$$
\left[L_{m}, Q_{n, \alpha}\right]=(n-m) Q_{m+n, \alpha}+\sum_{j=1}^{\infty} \Lambda^{2 j N} c_{m, n}^{j} Q_{m+n-2 j N, \alpha}
$$

Firstly, we prove nonrenormalization of $\left[L_{0}, Q_{n, \alpha}\right]$ using mathematical induction. The lowest dimensional correction to the commutators is $\Lambda^{2 N} Q_{-1, \alpha}$ hence the first step of induction 
is valid because the commutator of $L_{0}$ with $Q_{-1, \alpha}, \ldots, Q_{2 N-2, \alpha}$ does not have nonperturbative corrections. Assuming the induction hypothesis is valid for $Q_{-1, \alpha}, \ldots, Q_{n, \alpha}$ we calculate

$$
\begin{aligned}
{\left[L_{0}, Q_{n+1, \alpha}\right] } & =\frac{1}{n-1}\left[L_{0},\left[L_{1}, Q_{n, \alpha}\right]\right] \\
& =\frac{1}{n-1}\left[\left[L_{0}, L_{1}\right], Q_{n, \alpha}\right]+\frac{1}{n-1}\left[L_{1},\left[L_{0}, Q_{n, \alpha}\right]\right] \\
& =(n+1) \frac{\left[L_{1}, Q_{n, \alpha}\right]}{n-1}=(n+1) Q_{n+1, \alpha},
\end{aligned}
$$

where the first equality comes from the recursive definition of $Q_{n+1, \alpha}$, the second from Jacobi identity, the third from the induction hypothesis and the nonrenormalization of the Virasoro algebra and the last equality is again from the recursive definition of $Q_{n+1, \alpha}$. We show the absence of corrections to the remaining $[L, Q]$ commutators by commuting them with $L_{0}$ and then using Jacobi identity and the commutators we showed above to be nonrenormalized

$$
\left[L_{0},\left[L_{m}, Q_{n, \alpha}\right]\right]=\left[\left[L_{0}, L_{m}\right], Q_{n, \alpha}\right]+\left[L_{m},\left[L_{0}, Q_{n, \alpha}\right]\right]=(m+n)\left[L_{m}, Q_{n, \alpha}\right] .
$$

But the $\left[L_{m}, Q_{n, \alpha}\right]$ commutator is a linear combination of $Q_{k, \alpha}$ 's which are eigenvectors of the adjoint action of $L_{0}$ with eigenvalue $k$, whence the commutator is proportional to $Q_{m+n, \alpha}$ so all corrections to the commutator vanish. Let us show the absence of corrections to the $[L, R]$ commutator

$$
\left[L_{m}, R_{n}\right]=(n-m) R_{m+n}+\sum_{j=1}^{\infty} \Lambda^{2 j N} c_{m, n}^{j} R_{m+n-2 j N}+\sum_{j=1}^{\infty} \sum_{i=1}^{n+1} \Lambda^{2 j N} g_{i} d_{m, n}^{i, j} L_{m+n+i-2 j N} .
$$

We commute (A.4) with $Q_{l, \alpha}$ to get

$$
\begin{aligned}
{\left[Q_{l, \alpha},\left[L_{m}, R_{n}\right]\right]+\left[L_{m},\left[R_{n}, Q_{l, \alpha}\right]\right]+\left[R_{n},\left[Q_{l, \alpha}, L_{m}\right]\right] } & = \\
=\left[Q_{l, \alpha}, \sum_{j=1}^{\infty} \sum_{i=1}^{n+1} \Lambda^{2 j N} g_{i} d_{m, n}^{i, j} L_{m+n+i-2 j N}\right] & = \\
=\sum_{j=1}^{\infty} \sum_{i=1}^{n+1} \Lambda^{2 j N} g_{i}(m+n-l+i-2 j N) d_{m, n}^{i, j} Q_{m+n+i+l-2 j N, \alpha} & =0 .
\end{aligned}
$$

In simplifying (A.5) we used the $[L, Q]$ commutator which we proved above to be nonrenormalized and the $[R, Q]=0$ commutator. Clearly, the only way to satisfy the Jacobi 
identity (A.5) is that $d_{m, n}^{i, j}=0$. All $g_{i}$ dependent corrections vanish. The remaining corrections have the same algebraic structure as the corrections (A.5) to the $[L, Q]$ commutator so the nonrenormalization proof for that commutator works for the $[L, R]$ commutator as well.

It remains to consider the $\{Q, Q\}$ anticommutator. The nonperturbative corrections are proportional to $\epsilon_{\alpha \beta}$

$$
\begin{aligned}
\left\{Q_{\alpha, m}, Q_{\beta, n}\right\}= & -\epsilon_{\alpha \beta}(n-m) R_{m+n}-\epsilon_{\alpha, \beta} \sum_{j=1}^{\infty} \Lambda^{2 j N} c_{m, n}^{j} R_{m+n-2 j N} \\
& -\epsilon_{\alpha, \beta} \sum_{j=1}^{\infty} \sum_{i=1}^{n+1} \Lambda^{2 j N} g_{i} d_{m, n}^{i, j} L_{m+n+i-2 j N} .
\end{aligned}
$$

Consider the following Jacobi identity

$$
\begin{array}{r}
0=\left[L_{m},\left\{Q_{0, \alpha}, Q_{n, \beta}\right\}\right]+\left\{Q_{0, \alpha},\left[Q_{n, \beta}, L_{m}\right]\right\}-\left\{Q_{n, \beta},\left[L_{m}, Q_{0, \alpha}\right]\right\}= \\
-\epsilon_{\alpha, \beta} \sum_{j=1}^{\infty} \Lambda^{2 j N} R_{m+n-2 j N}\left[(n-m-2 j N) c_{0, n}^{j}+(m-n) c_{0, m+n}^{j}-m c_{n, m}^{j}\right] \\
-\epsilon_{\alpha, \beta} \sum_{j=1}^{\infty} \sum_{i=1}^{n+1} \Lambda^{2 j N} g_{i} L_{m+n+i-2 j N}\left[(n-m+i-2 j N) d_{0, n}^{i, j}+(m-n) d_{0, m+n}^{i, j}-m d_{n, m}^{i, j}\right] .
\end{array}
$$

Setting $m=0$ we get $c_{0, n}^{j}=0$ and $d_{0, n}^{i, j}=0$ unless $i=2 j N$. Substituting this back into (A.7) we see that all $c_{m, n}^{j}$ vanish and $d_{m, n}^{i, j}=0$ unless $i=2 j N$. To prove that the remaining corrections vanish we evaluate the $R, Q, Q$ Jacobi identity

$$
\begin{aligned}
{\left[R_{0},\left\{Q_{m, \alpha}, Q_{n, \beta}\right\}\right]+\left\{Q_{m, \alpha},\left[Q_{n, \beta}, R_{0}\right]\right\}-\left\{Q_{n, \beta},\left[R_{0}, Q_{m, \alpha}\right]\right\} } & = \\
{\left[R_{0},\left\{Q_{m, \alpha}, Q_{n, \beta}\right\}\right] } & = \\
-\epsilon_{\alpha, \beta}\left[R_{0}, \sum_{j>0} \Lambda^{2 j N} g_{2 j N} d_{m, n}^{j} L_{m+n}\right]=-\epsilon_{\alpha, \beta} \sum_{j>0} \Lambda^{2 j N} g_{2 j N}(m+n) d_{m, n}^{j} R_{m+n} & =0 .
\end{aligned}
$$

Hence, $d_{m, n}^{j} \equiv d_{m, n}^{2 j N, j}=0$ and the $\{Q, Q\}$ anticommutator is nonrenormalized. 


\section{References}

[1] R. Dijkgraaf and C. Vafa, "Matrix models, topological strings, and supersymmetric gauge theories," arXiv:hep-th/0206255.

[2] R. Dijkgraaf and C. Vafa, "On geometry and matrix models," arXiv:hep-th/0207106.

[3] R. Dijkgraaf and C. Vafa, "A perturbative window into non-perturbative physics," arXiv:hep-th/0208048.

[4] R. Argurio, G. Ferretti and R. Heise, "An Introduction to Supersymmetric Gauge Theories and Matrix Models," arXiv:hep-th/0311066.

[5] R. Dijkgraaf, M. T. Grisaru, C. S. Lam, C. Vafa and D. Zanon, "Perturbative computation of glueball superpotentials," arXiv:hep-th/0211017.

[6] F. Cachazo, M. R. Douglas, N. Seiberg and E. Witten, "Chiral rings and anomalies in supersymmetric gauge theory," arXiv:hep-th/0211170.

[7] K. Intriligator, P. Kraus, A. V. Ryzhov, M. Shigemori and C. Vafa, "On Low Rank Classical Groups in String Theory, Gauge Theory and Matrix Models," arXiv:hepth/0311181].

[8] N. Dorey, T. J. Hollowood, S. P. Kumar and A. Sinkovics, "Exact superpotentials from matrix models," JHEP 0211, 039 (2002) [arXiv:hep-th/0304138].

[9] V. Balasubramanian, J. de Boer, B. Feng, Y. H. He, M. x. Huang, V. Jejjala and A. Naqvi, "Multi-trace superpotentials vs. matrix models," Commun. Math. Phys. 242, 361 (2003) arXiv:hep-th/0212082.

[10] F. Cachazo, "Notes on supersymmetric $S p(N)$ theories with an antisymmetric tensor," arXiv:hep-th/0307063.

[11] P.L. Cho and P. Kraus, "Symplectic SUSY gauge theories with antisymmetric matter," Phys. Rev. D 54, 7640 (1996) [arXiv:hep-th/9607200.

[12] C. Csaki, W. Skiba and M. Schmaltz, "Exact results and duality for $S p(2 N)$ SUSY gauge theories with an antisymmetric tensor," Nucl. Phys. B 487, 128 (1997) arXiv:hep-th/9607210.

[13] P. Kraus and M. Shigemori, "On the matter of the Dijkgraaf-Vafa conjecture," JHEP 0304, 052 (2003) arXiv:hep-th/0304138.

[14] P. Kraus, A. V. Ryzhov and M. Shigemori, "Loop equations, matrix models, and $\mathcal{N}=1$ supersymmetric gauge theories," arXiv:hep-th/0304138.

[15] L. F. Alday and M. Cirafici, "Effective Superpotentials via Konishi Anomaly," arXiv:hep-th/0204119.

[16] J. Wess and B. Zumino, "Consequences of Anomalous Ward Identities," Phys. Lett. 37B, 95 (1971).

[17] C. Ahn and Y. Ookouchi, "Phases of $\mathcal{N}=1$ Supersymmetric SO/Sp Gauge Theories via Matrix Model," arXiv:hep-th/0302150. 
[18] A.Mironov et al. Phys.Lett. 252B (1990) 47; J.Ambjorn, J.Jurkiewicz and Yu. Makeenko, Phys.Lett. 251B (1990) 517; H.Itoyama and Y.Matsuo, Phys.Lett. 255B (1991) 202.

[19] A.Gerasimov, A.Marshakov, A.Mironov, A.Orlov et al. Nucl.Phys. B357 (1991) 565

[20] F. Cachazo, N. Seiberg and E. Witten, "Chiral Rings and Phases of Supersymmetric Gauge Theories," arXiv:hep-th/0303207.

[21] P. Svrcek, "Chiral Rings, Vacua and Gaugino Condensation of Supersymmetric Gauge Theories," arXiv:hep-th/0308037. 